\title{
The Formation of Fringes of Equal Chromatic Order across Double Clad Fiber in the Presence of High Power Lasers
}

\author{
Mona F. Omar*, Rania H. Abd El-Maksoud \\ Physics department, Faculty of Science, Ain Shams University, Cairo, Egypt \\ Email: "monafarouk67@hotmail.com
}

Received 5 November 2015; accepted 15 February 2016; published 18 February 2016

Copyright $@ 2016$ by authors and Scientific Research Publishing Inc.

This work is licensed under the Creative Commons Attribution International License (CC BY). http://creativecommons.org/licenses/by/4.0/

(c) (i) Open Access

\begin{abstract}
Multiple-beam fringes of equal chromatic order interferometry is a powerful technique to extract optical properties over a continuous range of spectrum. In this paper we develop a theory for the spectral shape of the fringes of equal chromatic order (FECO) that are formed across a double-clad fiber. The modified single term Sellmeier dispersion formula is used to construct the refractive index dispersion curves for the liquid, claddings, and core. Expressions for the paraxial optical path length for several rays passing through the fiber and the liquid are developed. The condition of bright fringe is applied to get an analytical expression for the spectral shape of the FECO across a double-clad fiber with an elliptical/circular inner cladding. A potential application of this theory is to determine the dispersion of Kerr constant of the claddings and the core of the fiber. To illustrate the theory and its application, simulation examples are provided and discussed.
\end{abstract}

\section{Keywords}

Interference, Physical Optics, Dispersion, Nonlinear Optics, Fringes of Equal Chromatic Order, Double-Clad Fibers

\section{Introduction}

Multiple-beam fringes of equal chromatic order (FECO) are formed when white light source illuminates a silvered air wedge. Each fringe corresponds to a specific optical path difference. However, since white light is used, these fringes are overlapped. To disperse the fringes, an auxiliary dispersion system is required. FECO interferometry is a powerful and sensitive tool in the field of metrology. Figure 1 shows an interferogram of

\footnotetext{
*Corresponding author.
}

How to cite this paper: Omar, M.F. and El-Maksoud, R.H.A. (2016) The Formation of Fringes of Equal Chromatic Order across Double Clad Fiber in the Presence of High Power Lasers. Optics and Photonics Journal, 6, 29-38. 


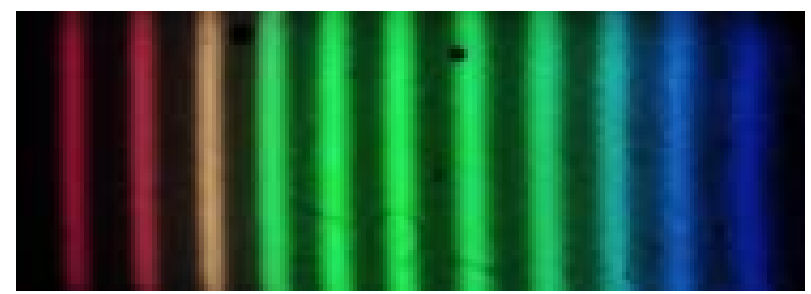

Figure 1. An interferogram of fringes of equal chromatic order that are formed by an air wedge.

FECO. Bennett and Eastman used multiple-beam FECO interferometry to determine accurately thin film thickness and surface roughness [1]. Lang and Scott used the fringes of equal chromatic order in reflection to study the resolution limits of topographical measurements [2]. Tabor and Winterton used FECO interferometry to measure the normal and retarded van der Waals Forces [3].

A great advantage of the FECO interferometry is that a single interferogram gives the required information to deduce the fiber optical parameters across the visible spectrum. Barakat et al. derived a mathematical expression for the shape of Fizeau fringes and FECO crossing an optical fiber with single circular cladding [4] [5]. Medhat et al. used the FECO to measure the dispersion curve of optical fibers with single cladding [6] [7]. In addition, Medhat et al. studied theoretically and experimentally the effect of the temperature and Gamma radiation on the dispersion curve of optical fibers with single cladding [8] [9].

A double-clad fiber (DCF) is an optical fiber that has a core and two cladding layers [10]. DCFs are available in several cladding geometries such as: rectangular, elliptical, circular with centered core, circular with shifted core, and D-shaped inner claddings [11]. Applications of DCFs include fiber lasers and endoscopy [11] [12]. Pal and Priye pointed out that profile perturbations such as refractive index dip in the core, which were typical features of fibers made by the MCVD technology, should be given a consideration in the design of double-clad single-mode fibers [13]. Abd El-Maksoud and Omar presented theoretical models for the fringe shift across DCFs that is inserted in a liquid air wedge and is illuminated with monochromatic light [14]. A methodology to reconstruct the linear and nonlinear terms of the refractive index profile of the DCF was outlined. Abd El-Maksoud et al. extended this work to include DCFs with graded-index inner cladding [15]. Accurate knowledge of the refractive index dispersion curve is essential in determining the information capacity that a fiber may transmit. To the best of authors' knowledge, there is no technique in the literature that addressed the problem of measuring the nonlinear refractive index dispersion of DCF. In this paper, we provide an understanding of the formation of the FECO across DCF and we discuss some of its applications. Potential applications include: 1) the determination of the dispersion curves of the linear and nonlinear refractive indices; 2) the determination of the refractive index dip. Section 2 presents the theory of the formation of the FECO across a DCF with an elliptical inner cladding. The effect of the nonlinear refractive index on the fringe shape is briefly elaborated. To illustrate the theory and its potential applications, simulation examples are presented. Finally, the conclusion is provided in Section 5 .

\section{Theory}

Fringes of equal chromatic order are produced when a liquid wedge interferometer is illuminated with a parallel beam of white light and is crossed with a spectrograph. The layout of the optical setup for producing the FECO across a DCF is shown in Figure 2. Since white light is used, the fringes that are formed by multiple reflections within the liquid wedge are overlapped. To disperse the fringes, an auxiliary dispersion system is required. The slit of the spectrograph is parallel to the edge of the wedge. Across the liquid region, fringe pattern is formed and consists of straight line fringes that are parallel to the edge of the wedge. Each fringe corresponds to a specific chromatic order $m$. The condition for bright fringe that is formed by transmitted the waves is [1]

$$
2 n_{L, \lambda} t=m \lambda
$$

where $t$ is the interferometric wedge thickness. $n_{L, \lambda}$ is the refractive index of the liquid at wavelength $\lambda$. Therefore, only specific wavelengths would satisfy Equation (1) for a specific $t$ giving rise to a chromatic order and therefore a chromatic fringe as shown in Figure 1. 


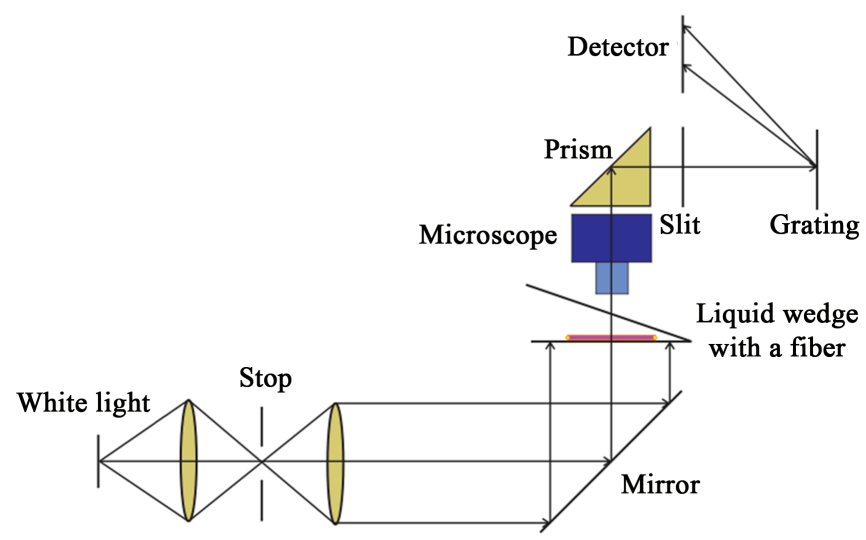

Figure 2. Optical setup layout for the formation and recording of FECO across a DCF that is inserted in a liquid wedge interferometer.

The step index DCF is inserted in a liquid wedge interferometer such that the fiber axis is perpendicular to the edge of the wedge. The axes are chosen such that the $\mathrm{x}$-axis is parallel to the edge of the wedge, the $\mathrm{y}$-axis is along the wedge thickness, and the fiber axis is along the z-axis. Figure 2 represents a cross section of a DCF with an inner elliptical cladding that has a semi axes $A$ (along the x-axis) and $B$ (along the y-axis). The outer cladding cross section is circular with radius $a_{C L 1}$. The core is circular with radius $a_{c}$ and its center is shifted along the $\mathrm{x}$-axis by a distance $\eta$. The refractive indices of the outer cladding, inner cladding and the core are $n_{C L 1, \lambda}, \quad n_{C L 2, \lambda}$ and $n_{c, \lambda}$, respectively. Medhat et al. used a modified single-term Sellmeier dispersion formula along with a correction technique to model the refractive index dispersion in optical fibers with a single cladding. Away from the absorption peaks, the refractive index maybe represented by [6]

$$
n_{\lambda}=\sqrt{\frac{2 h_{\lambda}+1}{1-h_{\lambda}}}
$$

where

$$
h_{\lambda}=A+\frac{B}{\lambda^{2}}+\frac{C}{\lambda^{4}}+\cdots
$$

where $A, B$, and $C$ are constants. Equation (3) along with Equation (2) maybe used to estimate the refractive index dispersion for the liquid, inner cladding, outer cladding, and the core.

Since the difference in the refractive indices of the liquid, outer cladding, inner cladding, and the core is small, refraction can be neglected in the calculation of the optical path length for rays passing through the DCF. The optical path length for ray $\mathrm{AB}$ that is passing through $R_{I}$ (defined by $A \leq\left|x_{I}\right| \leq a_{C L 1}$ ) is shown in Figure 3 and is given by

$$
O P L_{I, \lambda}=\left(t-2 y_{3, I}\right) n_{L, \lambda}+2 y_{3, I} n_{C L 1, \lambda}
$$

But:

$$
y_{3, I}=\left(a_{c L 1}^{2}-x_{I}^{2}\right)^{1 / 2} .
$$

Substituting Equation (5) in Equation (4) gives

$$
O P L_{I, \lambda}=n_{L, \lambda} t+2\left(a_{C L 1}^{2}-x_{I}^{2}\right)^{1 / 2}\left(n_{C L 1, \lambda}-n_{L, \lambda}\right) .
$$

The optical path length for ray CD that is passing through the liquid $R_{I I}$ (that is defined by is $\eta+a_{c} \leq x_{I I} \leq A$ and $-A \leq x_{I I} \leq \eta-a_{c}$ ) is shown in Figure 3 and is given by

$$
O P L_{I I, \lambda}=\left(t-2 y_{3, I I}\right) n_{L, \lambda}+2\left(y_{3, I I}-y_{2, I I}\right) n_{C L 1, \lambda}+2 y_{2, I I} n_{C L 2, \lambda} .
$$

But 


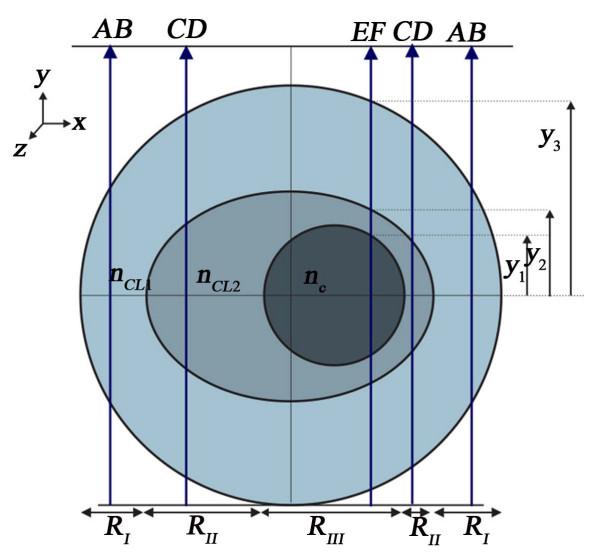

Figure 3. A cross section of the DCF with elliptical inner cladding and a shifted core.

$$
y_{2, I I}=B\left(1-\frac{x_{I I}^{2}}{A^{2}}\right)^{1 / 2} \text { and } y_{3, I I}=\left(a_{C L 1}^{2}-x_{I I}^{2}\right)^{1 / 2} .
$$

Substituting Equation (8) in Equation (7) gives

$$
O P L_{I I, \lambda}=n_{L, \lambda} t+2\left(a_{C L 1}^{2}-x_{I I}^{2}\right)^{1 / 2}\left(n_{C L 1, \lambda}-n_{L, \lambda}\right)+2 B\left(1-\frac{x_{I I}^{2}}{A^{2}}\right)^{1 / 2}\left(n_{C L 2, \lambda}-n_{C L 1, \lambda}\right) .
$$

The optical path length for ray EF that is passing through $R_{I I I}$ (defined by $\eta-a_{c} \leq x_{I I I} \leq \eta+a_{c}$ ) is shown in Figure 3 and is given by

$$
O P L_{I I I, \lambda}=\left(t-2 y_{3, \text { III }}\right) n_{L, \lambda}+2\left(y_{3, I I I}-y_{2, I I I}\right) n_{C L 1, \lambda}+2\left(y_{2, I I I}-y_{1, I I I}\right) n_{C L 2, \lambda}+2 y_{1, I I I} n_{c, \lambda} .
$$

But

$$
y_{1, I I I}=\left(a_{c}^{2}-\left(x_{I I I}-\eta\right)^{2}\right)^{1 / 2}, y_{2, I I I}=B\left(1-\frac{x_{I I I}^{2}}{A^{2}}\right)^{1 / 2} \text { and } y_{3, I I I}=\left(a_{C L 1}^{2}-x_{I I I}^{2}\right)^{1 / 2} \text {. }
$$

Substituting Equation (11) in Equation (10) gives

$$
\begin{aligned}
O P L_{I I I, \lambda} & =n_{L, \lambda} t \\
& +\left[2\left(a_{C L 1}^{2}-x_{I I I}^{2}\right)^{1 / 2}\left(n_{C L 1, \lambda}-n_{L, \lambda}\right)+2 B\left(1-\frac{x_{I I}^{2}}{A^{2}}\right)^{1 / 2}\left(n_{C L 2, \lambda}-n_{C L 1, \lambda}\right)+2\left(a_{c}^{2}-\left(x_{I I I}-\eta\right)^{2}\right)^{1 / 2}\left(n_{c, \lambda}-n_{C L 2, \lambda}\right)\right]
\end{aligned}
$$

The conditions of bright fringe with order $m$ across $R_{I}, R_{I I}$, and $R_{I I I}$ are given by

$$
\begin{gathered}
m \lambda=2 n t_{L}+4\left(a_{C L 1}^{2}-x_{I}^{2}\right)^{1 / 2}\left(n_{C L 1, \lambda}-n_{L, \lambda}\right) \\
m \lambda=2 n t_{L}+4\left[\left(a_{C L 1}^{2}-x_{I I}^{2}\right)^{1 / 2}\left(n_{C L 1, \lambda}-n_{L, \lambda}\right)+B\left(1-\frac{x_{I I}^{2}}{A^{2}}\right)^{1 / 2}\left(n_{C L 2, \lambda}-n_{C L 1, \lambda}\right)\right]
\end{gathered}
$$

$$
\begin{aligned}
m \lambda & =2 n t_{L} \\
& +4\left[\left(a_{C L 1}^{2}-x_{I I I}^{2}\right)^{1 / 2}\left(n_{C L 1, \lambda}-n_{L, \lambda}\right)+B\left(1-\frac{x_{I I I}^{2}}{A^{2}}\right)^{1 / 2}\left(n_{C L 2, \lambda}-n_{C L 1, \lambda}\right)+\left(a_{c}^{2}-\left(x_{I I I}-\eta\right)^{2}\right)^{1 / 2}\left(n_{c, \lambda}-n_{C L 2, \lambda}\right)\right] .
\end{aligned}
$$

Transforming the origin to $\left(2 n_{L, \lambda} t / m, 0\right)$ gives 


$$
\begin{gathered}
\lambda=\frac{4}{m}\left(a_{C L 1}^{2}-x_{I}^{2}\right)^{1 / 2}\left(n_{C L 1, \lambda}-n_{L, \lambda}\right) \\
\lambda=\frac{4}{m}\left[\left(a_{C L 1}^{2}-x_{I I}^{2}\right)^{1 / 2}\left(n_{C L 1, \lambda}-n_{L, \lambda}\right)+B\left(1-\frac{x_{I I}^{2}}{A^{2}}\right)^{1 / 2}\left(n_{C L 2, \lambda}-n_{C L 1, \lambda}\right)\right] \\
\lambda=\frac{4}{m}\left[\left(a_{C L 1}^{2}-x_{I I I}^{2}\right)^{1 / 2}\left(n_{C L 1, \lambda}-n_{L, \lambda}\right)+B\left(1-\frac{x_{I I}^{2}}{A^{2}}\right)^{1 / 2}\left(n_{C L 2, \lambda}-n_{C L 1, \lambda}\right)+\left(a_{c}^{2}-\left(x_{I I I}-\eta\right)^{2}\right)^{1 / 2}\left(n_{c, \lambda}-n_{C L 2, \lambda}\right)\right] .
\end{gathered}
$$

In the previous derivations, it is assumed that there are no fabrication defects in manufacturing the DCF. Equations (16)-(18) give the wavelength shift of the FECO across a DCF (with elliptical inner cladding) relative to the wavelength in the liquid region. Wavelength shift is attributed to the variation of optical thickness across the DCF. The contribution of the outer cladding, inner cladding, and the core to the wavelength fringe shift are half ellipses. The semi-axes of the half ellipses of the outer cladding, inner cladding, and core are $\left\{a_{C L 1}, 4\left(n_{C L 1, \lambda}-n_{L, \lambda}\right) / m\right\}$, $\left\{A, 4\left(n_{C L 2, \lambda}-n_{C L 1, \lambda} / m\right)\right\}$, and $\left\{a_{c}, 4\left(n_{c, \lambda}-n_{C L 2, \lambda} / m\right)\right\}$, respectively. The spectral fringe shift across a DCF with a circular inner cladding are obtained by using Equations (16)-(18) but with the substitution of $A=B=a_{C L 2}$.

\section{Simulation Example}

As an example, consider a step index DCF with dispersion curves as shown in Figure 4. The outer cladding and the centered core are circular and have radii of $62 \mu \mathrm{m}$ and $12 \mu \mathrm{m}$, respectively. The inner cladding is elliptical and has semi axes $A=45 \mu \mathrm{m}$ and $B=20 \mu \mathrm{m}$. The spectral fringe shape for the three fringes with chromatic orders of 1000, 1001, 1002 are shown in Figure 5. The shape of the three FECO across a circular inner and circular outer cladding with a displaced core $(\eta=15 \mu \mathrm{m})$ are shown in Figure 6. In this example, the DCF has an index profile that satisfies $n_{L, \lambda} \prec n_{C L 1, \lambda} \prec n_{C L 2, \lambda} \prec n_{c, \lambda}$. Therefore, the fringe shift is towards higher wavelengths.

To illustrate the spectral fringe shape across a DCF, consider a W DCF with claddings and core refractive index dispersion curves that are shown in Figure 7. The spectral fringe shape for an elliptical inner cladding with a centered core is shown in Figure 8. The Spectral fringe shape for a circular inner cladding with a displaced core is shown in Figure 9. In this example, the W DCF has an index profile that satisfies $n_{L, \lambda} \prec n_{C L 2, \lambda} \prec n_{C L 1, \lambda} \prec n_{c, \lambda}$. Therefore, the fringe shift is towards higher wavelengths for the outer cladding and core. However, the fringe shifts towards smaller wavelengths as it passes through the inner cladding.

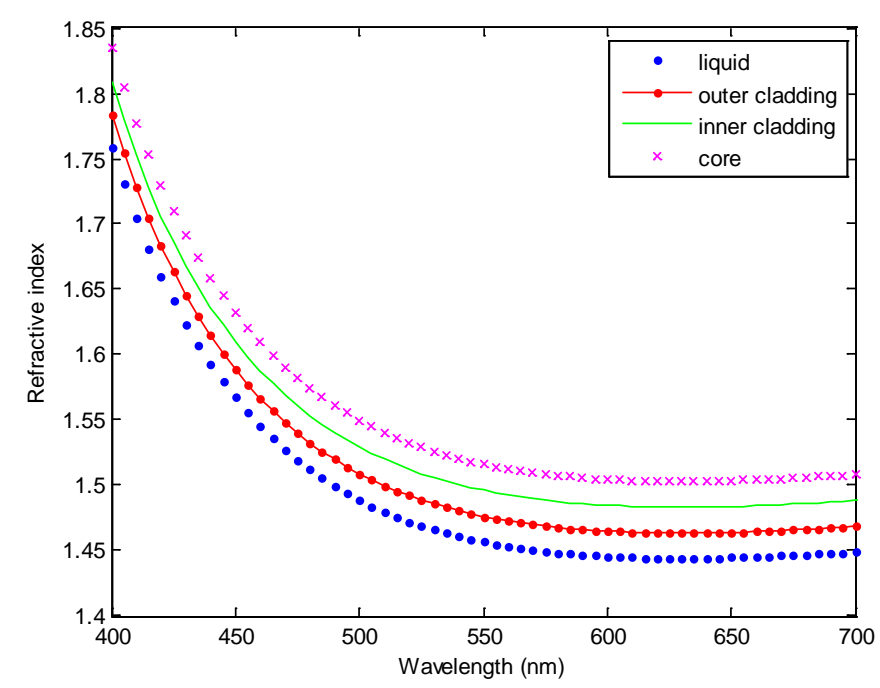

Figure 4. Refractive index dispersion curves for the liquid, outer cladding, inner cladding and the core. 


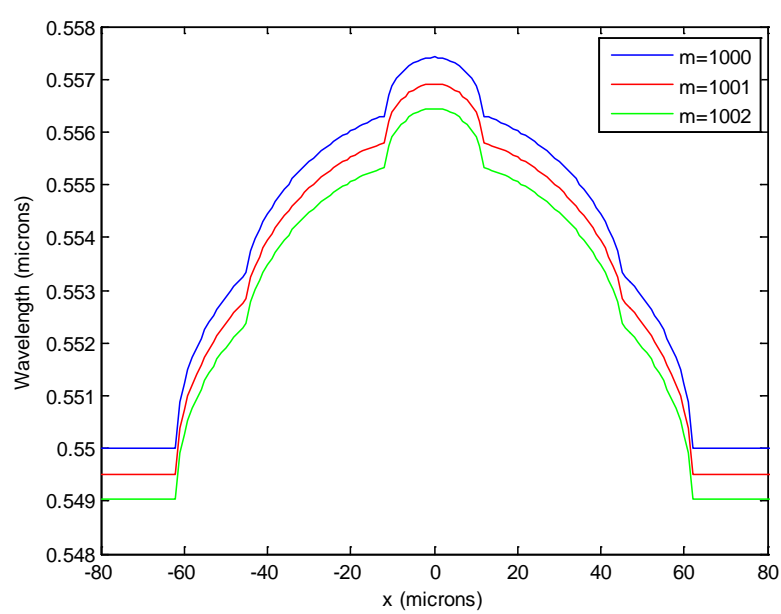

Figure 5. Spectral fringe shape across the DCF with elliptical inner cladding and centered core.

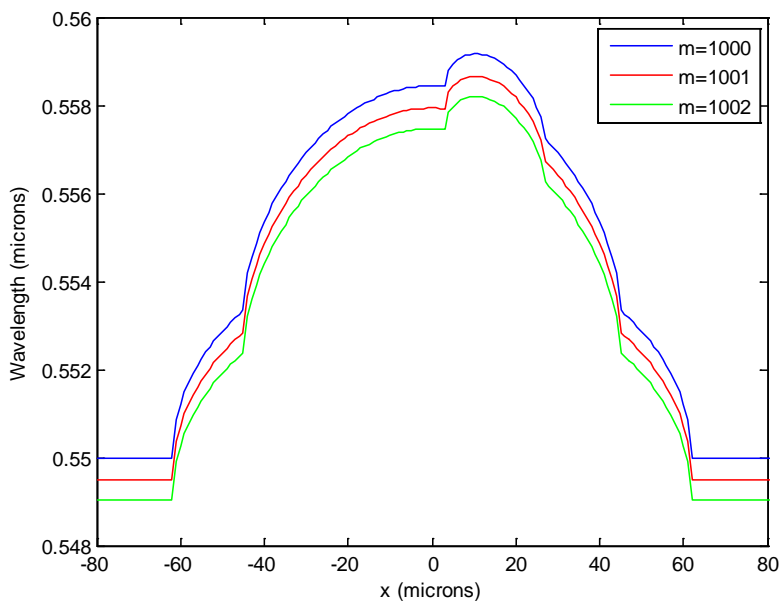

Figure 6. Spectral fringe shape for a DCF with circular inner cladding. The core is displaced from the DCF's center by 15 microns.

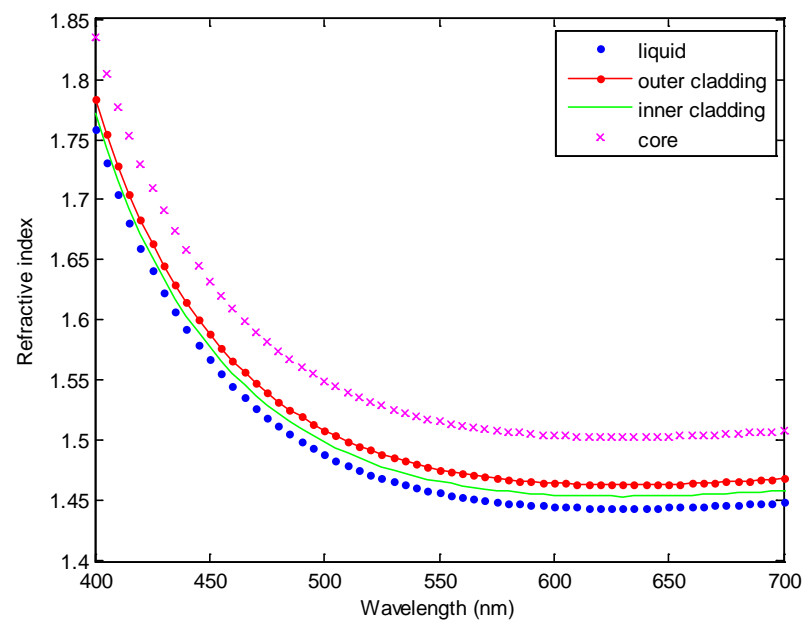

Figure 7. Refractive index dispersion curves for the liquid, outer cladding, inner cladding and the core of a W DCF (W DCF has an inner cladding with smaller refractive index than the outer cladding). 


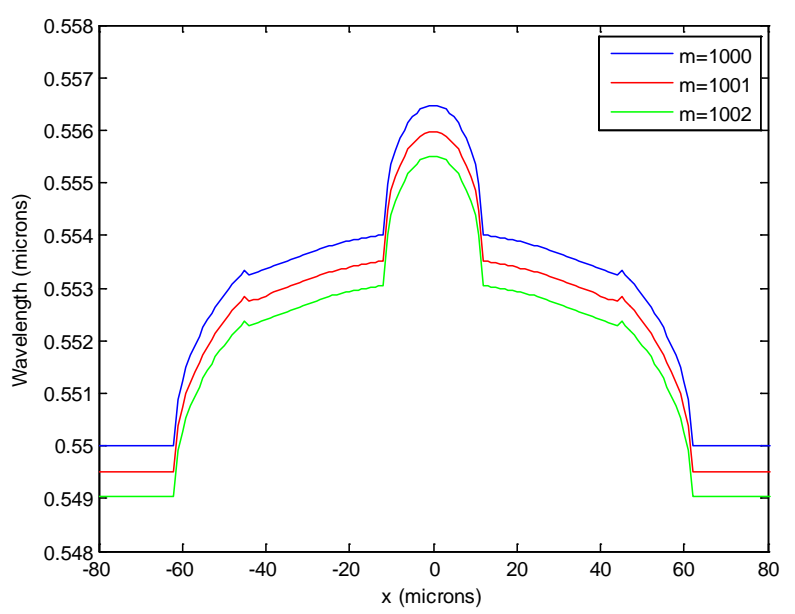

Figure 8. Spectral fringe shape for three orders across a W DCF with elliptical inner cladding and centered core.

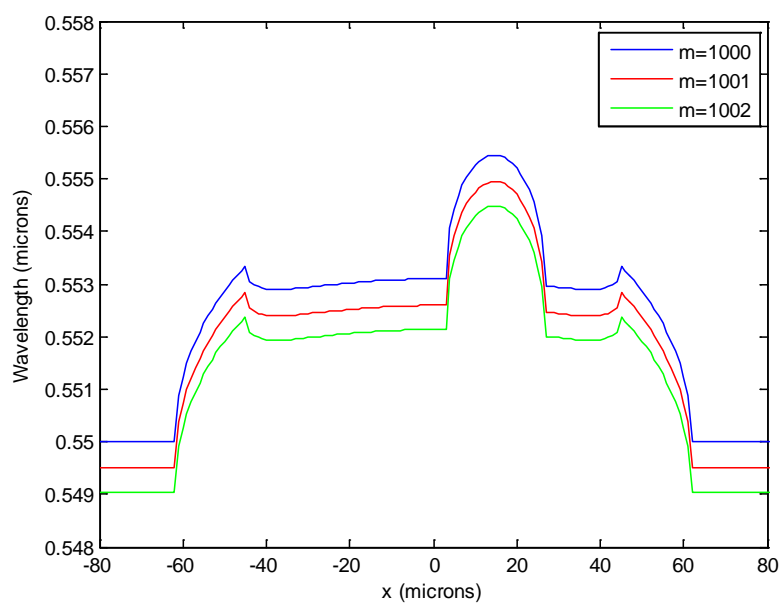

Figure 9. Spectral fringe shape for three orders across a W DCF with circular inner cladding. The core is displaced from the DCF's center by 15 microns.

\section{Potential Applications}

The first potential application of this theory is to measure experimentally the linear and nonlinear refractive index dispersion curves across double-clad fibers. This may be achieved by rewriting Equations (16)-(18) into

$$
\begin{gathered}
n_{C L 1, \lambda}=\frac{m \lambda}{4\left(a_{C L 1}^{2}-x_{I}^{2}\right)^{1 / 2}}-\left(\frac{t}{2\left(a_{C L 1}^{2}-x_{I}^{2}\right)^{1 / 2}}-1\right) n_{L, \lambda} \\
n_{C L 2, \lambda}=\frac{m \lambda-2 n_{L, \lambda} t-4\left(a_{C L 1}^{2}-x_{I I}^{2}\right)^{1 / 2}\left(n_{C L 1}-n_{L, \lambda}\right)}{B\left(1+\frac{x_{I I}^{2}}{A^{2}}\right)^{1 / 2}}+n_{C L 1, \lambda} \\
n_{c, \lambda}=\frac{m \lambda-2 n_{L, \lambda} t-4\left[\left(a_{C L 1}^{2}-x_{I I I}^{2}\right)^{1 / 2}\left(n_{C L 1, \lambda}-n_{L, \lambda}\right)-B\left(1-\frac{x_{I I I}^{2}}{A^{2}}\right)^{1 / 2}\left(n_{C L 2, \lambda}-n_{C L 1, \lambda}\right)\right]}{\left(a_{c}^{2}-\left(x_{I I I}-\eta\right)^{2}\right)^{1 / 2}}+n_{C L 2, \lambda} .
\end{gathered}
$$


In order to determine the linear refractive index dispersion curve experimentally, a reference wavelength is superimposed on the fringe pattern and a calibration equation is used to get the values of $(x, \lambda)$ for the claddings and the core. Then, these values are used to estimate the refractive index dispersion curve at each region. For example; to estimate the dispersion curve of the outer cladding; the liquid dispersion refractive index curve and the values of $\left(x_{I}, \lambda\right)$ are substituted at Equation (19). In the inner cladding, the values of $\left(x_{I I}, \lambda\right)$ and $n_{C L 1, \lambda}$ are substituted in Equation (20) to determine $n_{C L 2, \lambda}$. Same procedures are used for the core but with the corresponding values of $\left(x_{I I}, \lambda\right)$ and $n_{c, \lambda}$. If a high power laser is used, this would cause the refractive index to be dependent on the wavelength as well as the irradiance $\mathrm{E}$. This maybe represented for the outer cladding by the following equation

$$
n_{C L 1, \lambda}(E)=n_{C L 1, o, \lambda}+\Delta n_{C L 1, n, \lambda}(E)=n_{C L 1,0, \lambda}+n_{C L 1, n, \lambda} E
$$

where $n_{C L 1,0, \lambda}$ is Kerr constant and it is a function of the wavelength. The high irradiance changes the spectral refractive index profile across the fiber. This change in the refractive index profile causes the spectral fringe shape to shift. The amount of shift is a function of irradiance and wavelength. This concept maybe applied experimentally to estimate the dispersion curve for Kerr constant. To illustrate the concept, consider a DCF with circular inner cladding and a centered core. For simplicity, it is assumed that Kerr constant varies very slowly over the range of wavelengths that corresponds to orders 1000 and 1002, respectively. The assigned values for the change in the liquid, outer cladding, inner cladding and core are $0.0005,0.0004,0.0003$, and 0.0002 , respectively. The spectral fringe shape for low irradiance and for high irradiance is shown in Figure 10, respectively. The separation between the dotted and the solid lines gives the shift in the fringes due to the change in irradiance. The amount of shift is function of both the geometrical and optical properties.

So far, we assumed that there are no defects on the refractive index profile during the fabrication process. The second potential application is to measure fabrication defects. During the fabrication process, a refractive index dip in the core's refractive index may show up. The core's refractive index dip has a radius of $a_{d}$ and maybe represented by [16]

$$
n_{d}(r)=n_{d}(0)-\left\{n_{d}\left(a_{d}\right)-n_{d}(0)\right\}\left(\frac{r}{a_{d}}\right)^{\alpha}
$$

where $r$ is the radial distance and $\alpha$ is the refractive index profile parameter. For a DCF with an elliptical inner cladding and a centered core, the wavelength shift across the dip region that is defined by $-a_{d} \leq x_{d} \leq a_{d}$ is obtained by including the dip contribution term to Equation (18) and the substitution of $\eta=0$. Therefore, the wavelength fringe shift across the dip region is represented by

$$
\begin{aligned}
m \lambda= & 2 n t_{L}+4\left[\left(a_{C L 1}^{2}-x_{d}^{2}\right)^{1 / 2}\left(n_{C L 1, \lambda}-n_{L, \lambda}\right)+B\left(1-\frac{x_{d}^{2}}{A^{2}}\right)^{1 / 2}\left(n_{C L 2, \lambda}-n_{C L 1, \lambda}\right)\right. \\
& \left.+\left(a_{c}^{2}-x_{d}^{2}\right)^{1 / 2}\left(n_{c, \lambda}-n_{C L 2, \lambda}\right)+\left(a_{d}^{2}-x_{d}^{2}\right)^{1 / 2} \Delta n_{d, \lambda}-\Delta n_{d, \lambda} \int_{0}^{\left(a_{d}^{2}-x_{d}^{2}\right)^{1 / 2}}\left(\frac{r}{a_{d}}\right)^{\alpha} \mathrm{d} y\right]
\end{aligned}
$$

where $\Delta n_{d, \lambda}=n_{d, \lambda}(0)-n_{c, \lambda}$. To illustrate the contribution of the refractive index dip to the wavelength shift, consider a DCF that has a dip with $a_{d}=4$ microns, $\alpha=2$, and $\Delta n_{d, \lambda}=0.018$. The saddle contribution of the refractive index dip to the wavelength fringe shift is shown in Figure 11.

\section{Conclusion}

In this paper, the theory for the formation of the fringes of equal chromatic order across a double-clad fiber is presented. The double-clad fiber is perpendicular to the edge of the wedge. Each fringe as it passes through the fiber makes a wavelength shift with respect to the wavelength of the liquid fringe. Expressions for the wavelength fringe shift as a function of position along the DCF are developed. The developed expressions are applicable to DCFs that have elliptical or circular inner cladding with shifted or centered core. Furthermore, the 


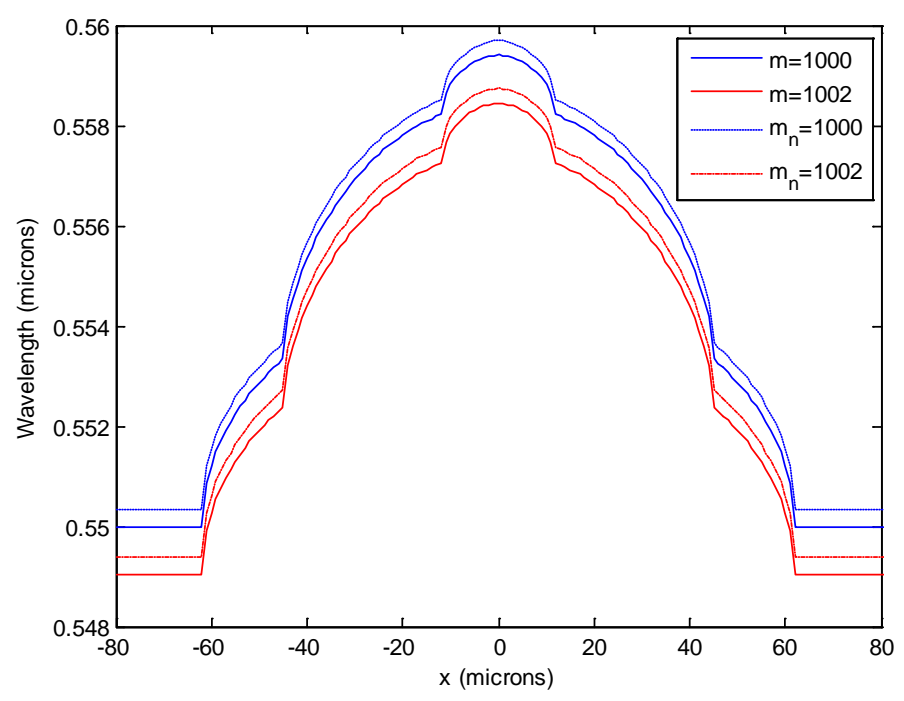

Figure 10. Spectral fringe shift for two orders across the DCF with inner circular cladding, and centered core. The outer cladding, inner cladding, and the core have radii of $62,45 \mathrm{mi}-$ crons, and 12 microns, respectively. The solid lines and dotted lines correspond to low and high irradiances, respectively.

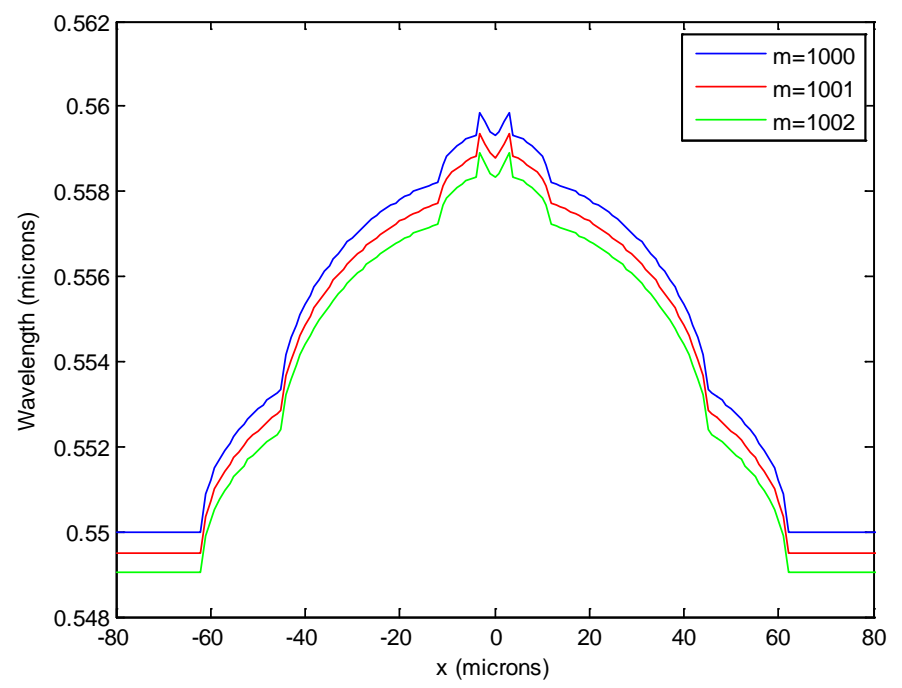

Figure 11. Spectral fringe shape across a DCF that has a refractive index dip around the center of the core. The DCF has an elliptical inner cladding and a centered core.

theory is extended to include the refractive index dip in DCF that has a centered core. To illustrate the theory, simulation examples are presented. The simulation examples show that the amount of wavelength fringe shift depends on the geometrical and the spectral optical properties of the fiber under study. Using a high power laser causes each order to be shifted. In other words, FECO interferometry is a very powerful technique to extract information about the linear and nonlinear dispersions of optical properties over a wide spectrum from a single interferogram. In addition, it may be used to measure the fabrication errors such as the refractive index dip. Future work includes the application of the theory to experimentally measure the linear and nonlinear refractive index dispersions of the optical parameters of DCF.

\section{References}

[1] Malcara, D. (1992) Optical Shop Testing. John \& Wiley Sons, Inc., USA. 
[2] Lang, J.E. and Scott, G.D. (1968) Resolution Limits in Multiple-Beam Interferometry. JOSA, 58, 81-83. http://dx.doi.org/10.1364/JOSA.58.000081

[3] Tabor, D. and Winterton, R.H.S. (1969) The Direct Measurement of Normal and Retarded Van der Waals Forces. Proceedings of the Royal Society of London, Series A, 312, 435-450. http://dx.doi.org/10.1098/rspa.1969.0169

[4] Barakat, N. (1971) Interferometric Studies on Fibers: Part I: Theory of Interferometric Determination of Indices of Fibers. Textile Research Journal, 41, 167. http://dx.doi.org/10.1177/004051757104100213

[5] Barakat, N. (1990) Interferometry of Fibrous Materials. Adam Hilger, Bristol and New York.

[6] Medhat, M., El-Zaiat, S.Y. and Omar, M.F. (1996) Determination of the Optical Properties of a Monomode Optical Fibre by Fringes of Equal Chromatic Order. Journal of Modern Optics, 43, 2205-2216. http://dx.doi.org/10.1080/09500349608232880

[7] Medhat, M., El-Zaiat, S.Y., Omar, M.F. and Barakat, N. (1997) Determination of the Material Dispersion and the Index Profile Parameters of a GRIN Optical Waveguide Applying White Light Interference Fringes. Journal of Modern Optics, 44, 461-472. http://dx.doi.org/10.1080/09500349708232914

[8] Medhat, M., El-Zaiat, S.Y., Radi, A. and Omar, M.F. (2002) Application of Fringes of Equal Chromatic Order for Investigating the Effect of Temperature on Optical Parameters of a GRIN Optical Fibre. Journal of Optics A: Pure and Applied Optics, 4, 174-179. http://dx.doi.org/10.1088/1464-4258/4/2/309

[9] Medhat, M., El-Zaiat, S.Y., Abdou, S.M., Radi, A. and Omar, M.F. (2002) Interferometric Determination of Gamma Radiation Effects on Optical Parameters of a GRIN Optical Fibre. Journal of Optics A: Pure and Applied Optics, 4, 485-490. http://dx.doi.org/10.1088/1464-4258/4/4/320

[10] http://en.wikipedia.org/wiki/Double-clad_fiber

[11] http://www.rp-photonics.com/double_clad_fibers.html

[12] Yelin, D., Bouma, B.E., Yun, S.H. and Tearney, G.J. (2004) Double Clad Fiber for Endoscopy. Optics Letters, 29, 2408-2410. http://dx.doi.org/10.1364/OL.29.002408

[13] Pal, B.P. and Priye, V. (1990) The Effect of an Axial Dip and Ripples in the Inner Cladding on the Leakage Loss of LP01 Mode in Depressed Index Clad Fibre. IEE Proceedings, 137, 311-314.

[14] Abd El-Maksoud, R.H. and Omar, M.F. (2011) Theory for the Measurement of the Linear and Nonlinear Refractive Indices of Double-Clad Fibers Using an Interferometric Technique. Applied Optics, 50, 5957-5965. http://dx.doi.org/10.1364/AO.50.005957

[15] Abd EL-Maksoud, R.H., Hillenbrand, M., Sinzinger, S. and Omar, M.F. (2012) Measuring the Refractive Index of Double-Clad Fibers Using an Interferometric Technique. DGaO-Proceedings.

[16] Barakat, N., El-Hennawi, H.A. and Sobeah, H.E. (1993) Multiple-Beam Interferometric Studies on Optical Fibres. Journal of Optics A: Pure and Applied Optics, 2, 419-428. http://dx.doi.org/10.1088/0963-9659/2/5/003 